\title{
Satisfacción laboral y compromiso organizacional: prueba de equivalencia de medición entre México y Estados Unidos
}

\author{
Job satisfaction and organizational commitment: Test of measurement equiva- \\ lence between Mexico and the United States
}
Daniel Arturo Cernas Ortiz ${ }^{1, *}$, Patricia Mercado Salgado ${ }^{1}$ y Filadelfo León Cázares ${ }^{2}$

${ }^{1}$ Universidad Autónoma del Estado de México, México

${ }^{2}$ Universidad de Guadalajara, México

Recibido el 27 de enero del 2016; aceptado el 20 de septiembre del 2016

Disponible en Internet el 19 de marzo de 2018

\section{Resumen}

El propósito de este trabajo fue analizar la equivalencia de medición de una escala de satisfacción laboral (Judge, Bono y Locke, 2000) y de una de compromiso organizacional (Allen y Meyer, 1990) entre la cultura mexicana y la estadounidense. Los datos se recolectaron por medio de un cuestionario auto administrado que se aplicó a una muestra no probabilística de estudiantes de maestría en ambas naciones (México = 287, Estados Unidos $=273$ ). Los resultados indicaron que las escalas analizadas no son invariantes entre las culturas. Una implicación de este hallazgo es que los estudios transculturales de satisfacción laboral y compromiso organizacional pueden reportar resultados inválidos en cuanto a la comparación directa de los niveles de los conceptos. Para evitarlo, se recomienda tomar ciertas precauciones en la preparación y en el análisis de los datos.

\section{Códigos JEL: M12, C38, C83}

Palabras clave: Equivalencia de medición, Satisfacción laboral, Compromiso organizacional, Cultura nacional, Diferencias entre México y Estados Unidos.

\footnotetext{
*Autor para correspondencia.

Correo electrónico: dcernasortiz@yahoo.com.mx (D.A.Cernas Ortiz)

La revisión por pares es responsabilidad de la Universidad Nacional Autónoma de México.
}

http://dx.doi.org/10.22201/fca.24488410e.2018.986

0186-1042/C 2018 Universidad Nacional Autónoma de México, Facultad de Contaduría y Administración. Este es un artículo Open Access bajo la licencia CC BY-NC-ND (http://creativecommons.org/licenses/by-nc-nd/4.0/). 


\begin{abstract}
The purpose of this study was to analysis the measurement invariance of a job satisfaction (Judge, Bono and Locke, 2000) and an organizational commitment (Allen and Meyer, 1990) scale between the Mexican and the U.S. cultures. Data were collected by means of a self-administered survey applied to a non-probabilistic sample of MBA students in both nations $($ Mexico $=287$, United States $=273)$. The results indicated that the scales under analysis were not invariant between the cultures. An implication of this finding is that cross-cultural studies of job satisfaction and organizational commitment might report invalid results concerning the direct comparison of the concepts' levels. In order to avoid this, it is recommended to take some precautions in the preparation and analysis of data.
\end{abstract}

JEL Classification: M12, C38, C83

Kew words: Measurement equivalence, Job satisfaction, Organizational commitment, National culture, Differences between Mexico and the United States of America

\title{
Introducción
}

Usualmente, la cultura se entiende como el conjunto de valores, creencias, normas y suposiciones compartidas por un grupo de personas en un espacio y un tiempo determinado (Minkov y Hofstede, 2012). Cada sociedad desarrolla una cultura que influye en la conducta $\mathrm{y}$, en consecuencia, en la vida de la gente que las integra. Diferentes sociedades evolucionan a través de distintas trayectorias históricas (Hofstede, Hofstede y Minkov, 2010). Por lo tanto, no es de sorprenderse que, entre sociedades, germinen divergencias en cuanto a las suposiciones fundamentales de los individuos sobre la vida y la existencia.

Para Octavio Paz (2009), México y Estados Unidos son dos países condenados a vivir uno junto al otro divididos por una profunda diferencia psíquica. Desde un paradigma funcionalista (véase Burrell y Morgan, 1979), trabajos de investigación con renombre internacional como los estudios de IBM (Hofstede et al., 2010; Minkov y Hofstede, 2012) y el proyecto GLOBE (House, Hanges, Javidan, Dorfman y Gupta, 2004) exhiben tales diferencias. Sin embargo, y contrario a lo que se publicita en medios masivos de comunicación y películas de Hollywood (véase Coatsworth y Rico, 1989), tales obras también indican que las diferencias entre ambas naciones no son tan pronunciadas como para alterar el significado de una variedad de conceptos relativos a las organizaciones.

No obstante, a menudo es necesario determinar si las formas de medir un concepto funcionan similarmente en entonos nacionales diferentes. Cierta evidencia indica que la gente de distintas sociedades no utiliza las escalas de medición en la misma manera. Por ejemplo, Smith (2004) encontró que, en comparación con la gente cuya cultura es predominantemente individualista (P. ej. EE.UU.), los ciudadanos de países con una cultura colectivista (P. ej. México) tienden a dar puntuaciones más altas y a utilizar menos el rango de opciones en sus respuestas a preguntas sobre valores. Si bien, entonces, las diferencias culturales podrían no alterar profundamente el significado de un concepto, sí podrían afectar el uso de las escalas de medición en una forma importante. Esta posibilidad es crítica debido a que la comparación directa de los niveles de un 
concepto podría carecer de sentido cuando los instrumentos de medición no son equivalentes entre los grupos sociales (Hair, Black, Babin, Anderson y Tatham, 2006; Wernsing, 2014).

La satisfacción laboral y el compromiso de los empleados con las organizaciones para las que trabajan son dos fenómenos organizacionales de gran relevancia en México y en Estados Unidos. Desde inicios del siglo XX, concretamente a partir de los estudios de Hawthorne (1924 - 1932), la satisfacción laboral ha sido una preocupación constante en varias áreas de la ciencia (Judge, Thoresen, Bono y Patton, 2001). Ligeramente más reciente, aunque no menos importante, es la atención que se ha dado al análisis del compromiso organizacional (Porter, Steers y Mowday, 2007). En ambos casos, el interés por indagar más sobre tales conceptos se debe, en gran parte, a que han sido asociados a las intenciones de renuncia y la rotación de personal, a la creatividad, el ausentismo y a una gran cantidad de fenómenos que afectan la efectividad de las organizaciones (Harrison, Newman y Roth, 2006; Raina y Britt Roebuck, 2016). Puesto que en el entorno económico integrado y competitivo de Norteamérica la efectividad organizacional es crucial para las empresas (Gazol Sánchez, 2010), el estudio de la satisfacción laboral y el compromiso organizacional siguen siendo relevantes y las comparaciones directas entre naciones cada vez más informativas. Con la finalidad de conocer la fiabilidad y la pertinencia de tales comparaciones, es necesario analizar la equivalencia de los instrumentos de medición de satisfacción y compromiso entre México y su vecino del norte.

En el contexto anterior, el objetivo del presente trabajo es analizar la equivalencia de una escala de medición de satisfacción laboral (Judge, Bono y Locke, 2000) y otra de compromiso organizacional (Allen y Meyer, 1990) entre México y Estados Unidos. Estas formas de medición son comúnmente utilizadas en la investigación y, por lo tanto, son pertinentes para los propósitos planteados en este documento. El análisis de equivalencia se lleva a cabo con una muestra de 287 y 273 estudiantes de maestría en administración, mexicanos y estadounidenses respectivamente. La estimación se realiza mediante modelamiento de ecuaciones estructurales, que es una técnica que toma en cuenta tanto la varianza de los conceptos como el error de medición (Byrne, 1998).

Los resultados de este trabajo contribuyen a la literatura sobre satisfacción laboral y compromiso organizacional en entornos internacionales. En resumen, se muestra que las escalas de medición de los conceptos analizados no son equivalentes entre México y Estados Unidos. También, los resultados indican que el significado que los participantes mexicanos atribuyeron a las cantidades de satisfacción laboral y de compromiso organizacional representadas en las escalas difirió del de sus contrapartes estadounidenses. Al parecer, la cultura de las naciones altera la percepción de las cantidades representadas en los intervalos de las escalas de medición. De tal suerte, mientras que para los mexicanos un puntaje de 4 en una escala tipo Likert de satisfacción laboral (o compromiso) podría significar estar "muy satisfecho", para un estadounidense ese mismo puntaje podría significar estar solo "satisfecho".

La organización del resto de este documento es como sigue. En la sección contigua se exponen sucintamente las diferencias culturales entre México y Estados Unidos de acuerdo al esquema de Hofstede. Después, se abordan brevemente los conceptos de satisfacción laboral y compromiso organizacional así como sus formas de medición. A continuación, se explica cómo es que la cultura nacional puede afectar la validez de los resultados de investigación sobre satisfacción laboral y compromiso organizacional cuando se hacen comparaciones directas entre países. Luego se detalla el método empleado en la investigación y se presentan 
los resultados. Este documento termina con la discusión de los hallazgos y con la presentación de algunas recomendaciones para investigadores interesados en las actitudes laborales a través de las fronteras internacionales. En la conclusión se resalta el valor de este trabajo para los estudios organizacionales.

\section{Diferencias culturales entre México y Estados Unidos}

Octavio Paz (2009) indica que México y Estados Unidos son dos versiones distintas de la cultura occidental. Para este autor, como herederos de las culturas española (católica) e inglesa (protestante) respectivamente, los dos países exhiben cosmovisiones diferentes que hacen que sus ciudadanos se comporten diferente, e incluso contradictoriamente. A este respecto, Riding (1985) observó que es posible que en ningún otro lugar del mundo existan dos naciones que viven lado a lado y que se conozcan y entiendan tan poco como México y Estados Unidos. Según Paz, y debido a sus diferencias, estas naciones mantienen una relación de amor y odio pues ambas imitan y a la vez repudian y desprestigian abiertamente algo de la otra. La ignorancia, la reticencia y la suspicacia que rodean a la vecindad entre México y la unión americana son complejas en muchos sentidos además del cultural. No obstante, tal complejidad parece sintetizarse en la referencia común que en México se hace a EE.UU. como "el otro lado" (algo extraño) y en EE.UU. a México como un "backyard" (traspatio o lugar de importancia secundaria)" (Coatsworth y Rico, 1989).

\section{México y Estados Unidos en términos de las dimensiones de Hofstede}

En los llamados Estudios de IBM, Hosftede (1980) indica que las naciones difieren culturalmente en cuatro dimensiones: evasión de la incertidumbre, masculinidad, distancia de poder, e individualismo. Más recientemente (Hofstede et al., 2010), este abanico se expandió incluyendo dos dimensiones más: orientación al corto plazo e indulgencia. En breve, evasión de la incertidumbre es el grado con que los integrantes de una sociedad sienten ansiedad y estrés ante lo incierto y, en general, prefieren lo cierto y lo concreto. Masculinidad es la prevalencia de valores como asertividad, competitividad y agresividad en la sociedad. Distancia de poder indica el grado con el que en un grupo social el poder se divide desigualmente y tal diferencial es aceptado y esperado. Individualismo denota un nivel bajo de cohesión grupal de tal suerte que cada individuo vela por sí mismo sin ser leal a algún grupo en particular. Orientación al corto plazo implica un enfoque de atención y energía en lo inmediato sin tomar muy en serio las consecuencias futuras. Indulgencia se refiere a la tendencia de las sociedades a permitirse la gratificación de deseos y placeres relacionados a la diversión y al goce de la vida.

Ciertamente, los estudios de Hofstede han recibido severas críticas. Entre otras cosas, por concebir a la cultura nacional como un fenómeno enteramente objetivo, además de ser simplistas en pretender capturar la complejidad del fenómeno con base en unas cuantas dimensiones abordadas mediante métodos nomotéticos (McSweeney, 2002). No obstante, si bien, los estudios de Hofstede son una aproximación a la complejidad de la cultura, tienen la gran ventaja de enfocarla con suficiente precisión y parsimonia como para comunicar a una gran audiencia los avances que, desde la perspectiva funcionalista, se realizan para desentrañar la "caja negra" del concepto de cultura nacional (Williamson, 2002). 
En sus estudios de 2010, Hofstede midió y cuantificó las seis dimensiones culturales por medio de un cuestionario aplicado a los trabajadores de la empresa IBM en 107 países. El autor y sus colegas procesaron las mediciones para obtener un índice por dimensión cultural. El índice da como resultado un ordenamiento (ranking). Entre más alta es la puntuación de un país en el índice, más prevalente es la dimensión cultural entre sus ciudadanos.

México y Estados Unidos presentan diferencias con respecto a las medidas de Hofstede et al. (2010). México es mucho más alto que Estados Unidos en evasión de la incertidumbre, distancia de poder, e indulgencia. Aunque la diferencia es menor, México también es más alto en masculinidad y orientación al corto plazo. A su vez, Estados Unidos es mucho más alto que su vecino en individualismo. La Figura 1 exhibe gráficamente estas diferencias. En la ilustración se muestra la distancia cultural global de Estados Unidos en relación a 32 países ${ }^{3}$. Como se observa (línea sólida), Estados Unidos es culturalmente más cercano a otras naciones anglosajonas (P. ej. Australia y Reino Unido) que a ciertos países de Europa oriental (Grecia y Rusia) y del extremo oriente asiático (P. ej. Singapur y Malasia).

En la Figura 2 se muestra el mismo conjunto de países que en la figura anterior (a excepción de Suecia), pero ahora México es la base de comparación. Como se observa, del conjunto de países incluidos, el más cercano a México es Brasil -lo que no causa sorpresa pues ambas son naciones latinoamericanas. Entre los más cercanos a México también están Grecia, Rusia y España, además de naciones del extremo oriente asiático como Taiwán y Corea del Sur. En la Figura 2, los países anglosajones y de la rivera del Mar Báltico son los más lejanos a México culturalmente hablando.

${ }^{3}$ Las puntuaciones globales se obtuvieron mediante la fórmula de Kogut y Singh (1988) con base en datos de Hofstede et al. (2010). Véase la Fórmula 1. Se tomaron en cuenta cuatro dimensiones: evasión de la incertidumbre, masculinidad, distancia de poder e individualismo

$$
K S_{i j}=\frac{1}{n} \sum_{d=1}^{n} \frac{\left(I_{i}^{d}-I_{j}^{d}\right)^{2}}{V^{d}}
$$

En la Fórmula 1, KS es la distancia cultural entre los países j e i. $i_{x}^{d}$ es el lugar de un país x en el índice de la dimensión cultural d, Vd es la varianza del índice para la dimensión d, y n es el número de dimensiones culturales consideradas en el cálculo. En este documento, el conjunto de países involucrados sólo es ilustrativo. Por lo tanto, se concede que la cercanía cultural entre México y EE.UU. puede cambiar si las puntuaciones globales se obtienen mediante el análisis de otro conjunto de países. 


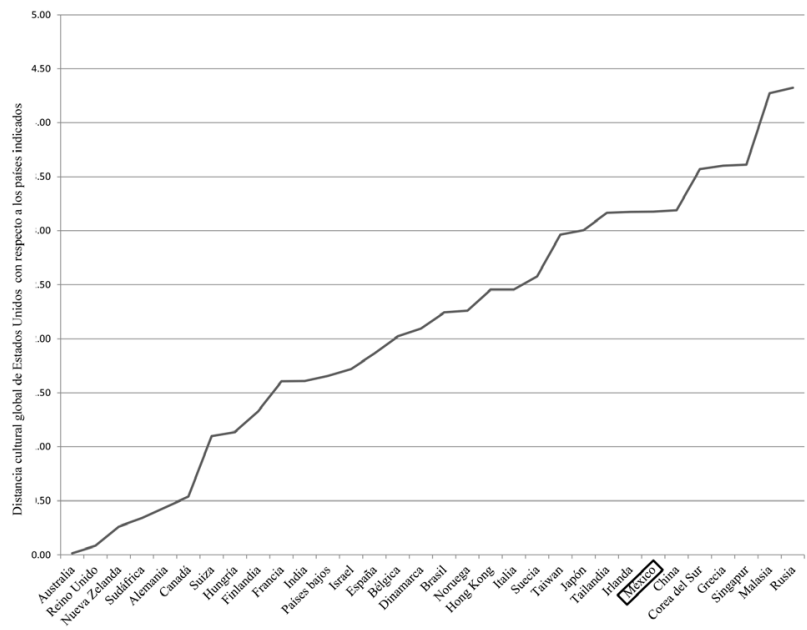

Figura 1. Distancia cultural entre Estados Unidos y 32 países Fuente: Elaboración propia

En las figuras 1 y 2 se aprecia la lejanía cultural entre México y Estados Unidos. Las distancias, en turno, hacen sospechar que las diferencias culturales podrían afectar el funcionamiento de los instrumentos (escalas de medición) que a menudo se utilizan para medir una gran variedad de fenómenos concernientes al comportamiento humano. No se omite recordar que tales instrumentos a menudo son creados en EE.UU. y aplicados en México (y en otros países) mediante traducciones de cuya confiabilidad hay poca evidencia documentada.

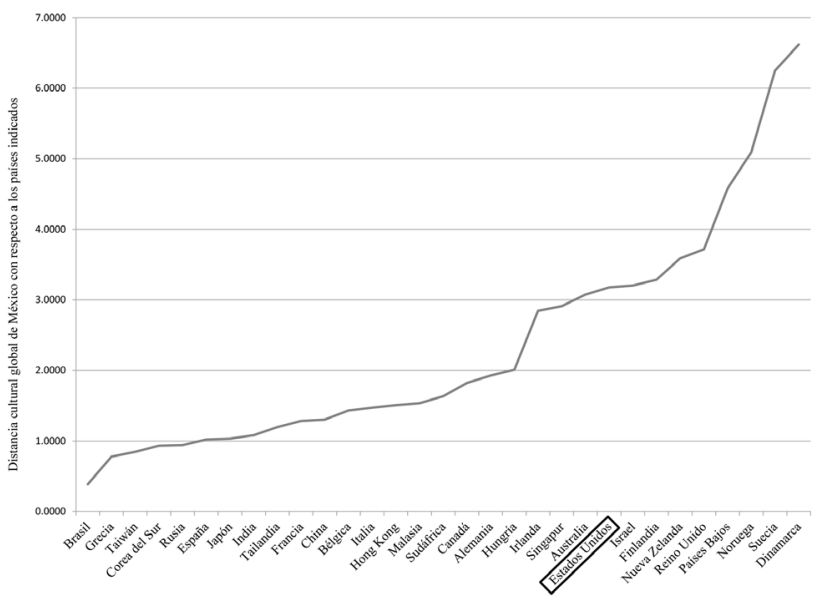

Figura 2. Distancia cultural entre México y 31 países Fuente: Elaboración propia 


\section{Satisfacción laboral}

La satisfacción laboral es importante debido a su supuesta relación con la productividad del personal. Locke (1969) define a este concepto como el sentimiento positivo hacia el trabajo que resulta de una evaluación de sus características. En su tratado filosófico y sociológico sobre los estudios organizacionales, Burrell y Morgan (1979) indican que los beneficios del estudio de la satisfacción han entrado en el reino de la mitología administrativa. Los autores argumentan que desde los estudios de Hawthorne, se ha creído casi ciegamente que la satisfacción laboral es importante debido a que promueve la productividad en las empresas. Entre los psicólogos industriales, hubo quienes incluso consideraron a la relación entre satisfacción y desempeño laboral como el Santo Grial de los estudios organizacionales (Landy, 1989).

Sin embargo, la verdadera relevancia del concepto así como su relación con el desempeño laboral siguen siendo asuntos discutidos. Un meta-análisis influyente por Iaffaldano y Muchinsky (1985) mostró que la correlación entre satisfacción y desempeño en el trabajo es relativamente baja $(\mathrm{r}=0.17)$, poniendo en duda la relevancia práctica de estudiar a la satisfacción para elevar la productividad. Posteriormente, Judge et al. (2001) mostraron que la correlación entre satisfacción y desempeño puede ser tan alta como 0.25 , reavivando la discusión sobre la importancia del concepto de satisfacción.

Renovado el entusiasmo, diversos investigadores han indagado sobre la dirección de la causalidad entre satisfacción y desempeño. De hecho, y en franca oposición a la teoría del comportamiento planificado y a los modelos de la acción razonada, tal parece que la satisfacción (actitud) depende más del desempeño en el trabajo (comportamiento) que vice-versa. En su teoría de la disonancia cognoscitiva, Festinger (1957) fue de los primeros en reconocer esta posibilidad. Para esta teoría, la actitud es la consecuencia de la conducta, pues las personas siempre buscamos consistencia entre nuestros comportamientos y actitudes. Así, por ejemplo, cuando alguien recibe una recompensa laboral, se da cuenta de que está tendiendo un alto desempeño, y esto, en turno, le hace pensar que su desempeño (comportamiento) se debe a que le gusta lo que hace (la actitud hacia el trabajo). Evidencias basadas en la teoría de la expectación (Vroom's expectancy theory, 1964) respaldan tales argumentos, pues indican que el alto desempeño conduce a recompensas y éstas producen satisfacción. Otros trabajos como los de Wright y Staw (1999) y los de Peterson, Park, Hall y Seligman (2009) aun sugieren que la satisfacción laboral es un reflejo de la felicidad individual y la satisfacción con la vida como rasgos inherentes a la personalidad. Aunque interesante, esta línea de investigación es un tanto extrema pues implica que la productividad tiene poco que ver con la satisfacción en el trabajo. Al parecer, la gente feliz estaría mayormente satisfecha independientemente de sus resultados laborales (Salanova, Martínez y Llorens, 2005). Aún con todo, al día de hoy, la investigación sobre satisfacción laboral continúa haciéndose, pues varios estudios indican que tal concepto existe, que tiene significado en las culturas occidentales y que de una forma u otra sí podría tener un efecto en los resultados del trabajo (Harrison et al., 2006; Judge y Klinger, 2008).

El concepto en mención ha sido medido en múltiples formas. Desde finales de los 70s se ha documentado la gran variedad de instrumentos existentes para medir la satisfacción laboral (véase O’Connor, Peters y Gordon, 1978). Algunas de las escalas más utilizadas son aquéllas que, como el JDI (por el acrónimo en inglés de Job Descriptive Index) o el JSS (por el acrónimo en inglés de Job Satisfaction Survey), miden la satisfacción en sus varias facetas (Gillespie, et al., 2016). No obstante, la satisfacción global con el trabajo es diferente de la suma de la 
satisfacción con las facetas del mismo (Bowling, Hendricks y Wagner, 2008). En consecuencia, las medidas globales suelen ser las más adecuadas para estudiar las relaciones del concepto bajo análisis con otras actitudes como el compromiso organizacional (véase Curry, Wakefield, Price y Mueller, 1986). Una de las medidas globales más utilizadas ha sido el índice de Brayfield y Rothe (1951), sobre todo sus versiones cortas como la de seis reactivos propuesta por Curry et al. (1986) o la de cinco propuesta por Judge et al. (2000). La evidencia indica que, sobre todo la medida más corta, es válida y confiable siendo utilizada muy comúnmente en los estudios que abordan la satisfacción global en el trabajo (Judge y Klinger, 2008).

\section{Compromiso organizacional}

Después de la satisfacción, el compromiso organizacional es quizá la actitud laboral más estudiada en el mundo entero. Investigaciones tempranas sobre este concepto pueden rastrearse a los postulados de Etzioni (1961), quien desde un punto de vista sociológico resaltó la importancia y los beneficios del compromiso hacia la organización en la época de la posguerra. Sin embargo, una de las primeras definiciones ampliamente difundidas del término se debe a Porter, Mowday y Steers (1982), quienes concibieron el compromiso como la fuerza relativa de la identificación del personal, y su involucramiento, con una organización en particular. Aunque Porter y sus colegas han sido influyentes en el tema, fueron Allen y Meyer (1990) quienes lanzaron el concepto a la fama mundial con la postulación de su modelo tridimensional de compromiso: afectivo, normativo y de continuidad. Esta conceptualización fue, y aún es, bien recibida por los investigadores en la materia. No obstante, la dimensión afectiva en solitario se ha reafirmado como la forma más común de estudiar el compromiso. A diferencia de las dimensiones normativa y de continuidad, es la única cuyo objeto de evaluación interna (la actitud per sé) es la organización (una entidad abstracta) y no el (posible) abandono de la misma (un comportamiento concreto) (Solinger, van Olfen y Roe, 2008).

Al igual que la satisfacción, el compromiso organizacional es una actitud debatida. Por un lado, y con base en teorías como el comportamiento planificado y el intercambio social, se supone que un alto compromiso hacia la organización promueve comportamientos positivos (P. ej. ciudadanía e innovación) y reduce los negativos (P. ej. acoso laboral e indiferencia) (Gill, Meyer, Lee, Shin y Yoon, 2011). No obstante, esta lógica también es susceptible de operar en reversa. Esto es, las recompensas obtenidas por mostrar comportamientos positivos pueden elevar, y quizá hasta producir, el compromiso. Investigaciones sobre el soporte organizacional percibido apoyarían esta lógica. Después de todo, un alto desempeño en el trabajo conlleva un mayor cuidado del empleado por parte de la organización, y hasta cierta condescendencia con él/ella (Rhoades y Eisenberger, 2002). Tal cuidado eleva el soporte percibido y éste, a su vez, el compromiso. Independientemente de la causalidad entre el compromiso y sus antecedentes, estudios en diferentes entornos nacionales han puesto de relieve que, al igual que la satisfacción laboral, el compromiso es un concepto que existe y que tiene significado en varias culturas (Gill et al., 2011; Meyer, Stanley, Herscovitch y Topolnytsky, 2002).

Congruente con el predominio conceptual del compromiso organizacional afectivo de Allen y Mayer (1990), la forma más común de medir el compromiso es mediante la escala desarrollada por los mismos autores. Esta escala es más sucinta que otras medidas alternativas como el OCQ (Organizational Commitment Questionnaire) de Mowday, Steers y Porter (1979), y ha 
sido reportada como altamente confiable $(\alpha>0.70)$ en una gran cantidad de estudios (véase Meyer et al., 2002; Vandenberghe, Bentein, Panaccio, 2014). Los reactivos originales eran ocho, aunque para mayor brevedad, posteriormente, se redujeron a seis (Meyer, Allen y Smith, 1993). En cualquier caso, existe evidencia de la validez de constructo del instrumento de Allen y Meyer en Estados Unidos y Canadá, pues está fuertemente relacionado con el OCQ de de quince reactivos $(\mathrm{r}>0.05)$ y con otras medidas reportadas en la literatura sobre el tema (Allen y Meyer, 1996; Meyer et al., 2002).

\section{Influencia de la cultura en la validez de los resultados de investigación}

De acuerdo con Campbell y Stanley (1963), la validez interna se entiende como la ausencia de explicaciones (o hipótesis) alternativas para la relación experimental entre causas y efectos, o para la correlación entre dos variables en un estudio no experimental. Existen múltiples amenazas a este tipo de validez (véase Cook, Campbell y Peracchio, 1990), teniendo cada una de ellas el potencial de menguar la credibilidad de los resultados obtenidos y de las conclusiones que de los mismos puedan hacerse. En los estudios no experimentales, una de las amenazas más preocupantes es el error de medición y sus dos componentes: el aleatorio y el sistemático (Bagozzi y Yi, 1991).

El error sistemático es particularmente serio porque es una explicación alternativa directa para la covariación observada entre dos conceptos. A menudo, tal error se entiende como un sesgo (o conjunto de sesgos como el halo, la deseabilidad social, la condescendencia y la lenidad) que altera (hacia puntuaciones altas o bajas) las respuestas a las preguntas que se incluyen en un cuestionario (Fiske, 1982). Cuando en la aplicación de un instrumento de medición existe un error sistemático alto, los constructos involucrados exhibirán una covariación (o correlación) alta debido a que todas las preguntas se contestaron con puntuaciones similares independientemente de su contenido (Bagozzi, Yi y Phillips, 1991; Podsakoff y Organ, 1986; Podsakoff, MacKenzie, Lee y Podsakoff, 2003). Sobra decir que la relación detectada será espuria.

Los sesgos de condescendencia y lenidad son particularmente susceptibles al efecto de la cultura nacional. De acuerdo con Podsakoff et al. (2003), la condescendencia (sí a todo) se refiere a la propensión de los respondientes a estar de acuerdo con los reactivos de una escala independientemente de su contenido. La lenidad, por su parte, es la propensión individual a otorgar puntuaciones indebidamente altas cuando el cuestionario se refiere a alguien (o algo) a quien se conoce o agrada. Interesantemente, ambos sesgos parecen implicar un "querer quedar bien" subconsciente con algo o alguien, o bien la evasión de alguna confrontación real o imaginaria con esa entidad. Puesto que la cultura engloba creencias, normas y suposiciones con respecto a las relaciones entre los individuos y la armonía social (Smith, 2004), tanto la lenidad como la condescendencia podrían ser más proclives a presentarse en unas culturas que en otras.

En el caso de México y Estados Unidos, es posible que las escalas de medición de satisfacción y compromiso funcionen de manera diferente debido a influencias culturales. Se ha documentado que el sesgo de condescendencia es más prevalente en sociedades altas en evasión de la incertidumbre, distancia de poder y colectivismo (Marín, Gamba y Marín, 1992; Smith, 2004). Este mismo perfil cultural podría pronunciar el sesgo de lenidad. La gente de culturas altas en evasión de la incertidumbre es proclive a dar puntuaciones elevadas a los 
reactivos de un cuestionario con la finalidad de minimizar la posibilidad de conflictos y la ansiedad e incertidumbre que éstos pueden generar. En las sociedades altas en colectivismo y distancia de poder los individuos pueden ser propensos a otorgar puntuaciones más positivas de lo que deberían debido a que ello favorece la armonía al interior del grupo y la deferencia hacia personas con una posición social probablemente superior. Aunado a esto, cuando una cultura es alta en indulgencia y orientación al corto plazo, la gente tiende a recordar más cosas positivas que negativas al momento de contestar una encuesta (Hofstede et al., 2010). Este efecto conocido como sesgo de disponibilidad eleva las puntuaciones artificialmente (Ruder y Bless, 2003). Dado que México es más alto que EE.UU. en la mayoría de las dimensiones culturales mencionadas, el sesgo de condescendencia es más alto en México (Marín et al., 1992) y probablemente el de lenidad también. Con base en los argumentos anteriores se postula siguiente hipótesis.

H. La escala de satisfacción laboral de Judge et al. (2000) y la escala de compromiso organizacional de Allen y Meyer (1990) no son equivalentes entre México y Estados Unidos.

\section{Método de investigación}

Este estudio cuantitativo y no experimental (de la equivalencia de las escalas indicadas entrUn total de 583 estudiantes de maestría con experiencia laboral participaron en la investigación. Después de eliminar las respuestas incompletas (4\% aprox.) la muestra final contenía 560 observaciones. Mediante un muestreo no probabilístico, los datos fueron recolectados en una universidad del centro de México $^{4}(n=287)$ y en una del suroeste de Estados Unidos ${ }^{5}(273)$.

Los estudiantes fueron contactados por profesores de las universidades involucradas y la participación fue voluntaria. Es importante notar que, aunque estas muestras son ilustrativas más que totalmente representativas de las sociedades a las que pertenecen, existen razones para presumir su adecuación a los propósitos de esta investigación. Locke (1986) indica que para que una muestra sea representativa de un universo, ésta debe exhibir las características esenciales de los elementos de ese universo. En este caso, todos los estudiantes de maestría considerados tienen experiencia laboral directamente relevante a la formación y al cambio de actitudes en el trabajo (en el siguiente párrafo se muestra la evidencia). También, debido a que son oriundos de los países bajo análisis, están tan imbuidos en la cultura de sus países como la mayoría de los trabajadores en tales sociedades. Como dato adicional, Harrison et al. (2006) encontraron evidencia meta-analítica (con una gran cantidad de muestras de trabajadores) que la correlación entre satisfacción laboral y compromiso organizacional es de 0.60 aproximadamente. En las muestras examinadas en este trabajo, la correlación entre tales constructos está muy cerca de este parámetro (véase Tabla 1 más adelante). Claramente, los datos utilizados en esta investigación se comportan como muchas otras muestras estudiadas anteriormente. En turno, esto brinda certeza sobre su representatividad y adecuación a los propósitos de esta investigación. Sin embargo, se reconoce que los resultados de este estudio pueden tener una generalidad limitada. Con respecto a que dentro de un país existe variedad cultural y que, por lo tanto, los resultados obtenidos con una muestra de una región no serían generalizables a otras, Minkov y Hofstede (2012) encontraron que, tanto en México y como en EE.UU., las diferencias culturales entre regiones son mucho menores que las diferencias culturales que, en general, existen entre los dos países.

\footnotetext{
${ }^{4}$ Universidad Autónoma del Estado de México

${ }^{5}$ Universidad del Norte de Texas
} 
Las muestras exhiben pequeñas diferencias, pero, por la mayor parte, son muy similares en términos de la demografía de los participantes. El número de mujeres fue un poco más alto en México (55.4\% mujeres, $44.6 \%$ hombres) que en EE.UU. (48.5\% mujeres, 51.5\% hombres). La mayoría de los participantes eran empleados de tiempo completo (72.4\% México, $59.9 \%$ EE.UU.), aunque la base de datos estadounidense contenía un mayor número de desempleados que su contraparte mexicana (3.8 \% México, $14.6 \%$ Estados Unidos). La edad promedio fue de 31.32 (d. e. = 5.77) años entre los mexicanos y de 29.34 (d. e. $=7.24$ ) entre los estadounidenses. En ambos casos, los estadísticos de asimetría fueron inferiores a (+-) 2, aunque los de kurtosis fueron superiores a los parámetros recomendados (+ - 2 ó 3). No obstante, las desviaciones de la distribución normal en cuanto a edad no afectan significativamente los resultados de este estudio ya que ésta no es una variable central en el análisis. No se recolectaron datos de ingreso porque esa pregunta fue considerada como sensitiva por las autoridades de la universidad estadunidense.

Los datos se recolectaron por medio de una encuesta auto-administrada. La tasa de respuesta fue alta porque los cuestionarios se aplicaron durante las horas de clase. Amablemente, se ofreció a los estudiantes la posibilidad de compartirles los resultados de la investigación como incentivo para responder honestamente al cuestionario. En todo caso se emplearon mecanismos para asegurar el anonimato de las respuestas, y por lo tanto, evitar sesgos consientes de respuesta como la deseabilidad social. Por ejemplo, en los cuestionarios no se pidieron nombres ni ningún dato que permitiera la identificación de los respondientes. También, se pidió que los profesores no estuvieran presentes en la sesión de recolección de datos y se dejo claro que la información sería estudiada en agregado y, por lo tanto, no habría juicios sobre individuos en particular (P. ej. qué tan satisfecho o comprometido está alguna persona en específico).

En concordancia con los propósitos de este trabajo, la satisfacción laboral y el compromiso organizacional se midieron con las escalas que se indicaron anteriormente: Judge et al. (2000) y Allen y Meyer (1990), respectivamente. En ambos casos, los reactivos se formatearon en una escala tipo Likert de siete grados con ancla verbal en 1 (muy en desacuerdo) y 7 (muy de acuerdo). Las oraciones "Yo realmente disfruto mi trabajo" y "Estaría feliz de pasar el resto de mi carrera en esta organización" ejemplifican las escalas de satisfacción y compromiso respectivamente. Los autores de las escalas reportan estimados de consistencia interna de 0.89 para la satisfacción y de 0.87 para el compromiso. Ambas escalas son unidimensionales.

Para la aplicación de la encuesta en México, las escalas en cuestión se tradujeron al español de su original en inglés. Después, una persona ajena al proyecto retradujo las preguntas a su idioma inicial. Esta re-traducción se comparó con la versión inglesa de las escalas y las discrepancias detectadas se corrigieron hasta que los reactivos se consideraron equivalentes en ambos idiomas. En todo caso, se empleó un proceso descentrado por medio del cual la traducción se hizo a significados comparables no literales.

\section{Análisis}

En aras de tener un primer acercamiento a los datos, se calculó la confiabilidad de los constructos se satisfacción laboral y compromiso organizacional por medio del método de consistencia interna (también conocido como Alpha de Chronbach). A continuación, se calcularon las medias de los constructos para las muestras de ambos países y se determinó si existían diferencias significativas entre ellas por medio de una prueba $t$ de Student a dos vías (two way). 
Posteriormente, la validez de los constructos, así como la equivalencia de las escalas a través de las culturas analizadas (la hipótesis de este trabajo), se probaron mediante análisis factorial confirmatorio (AFC) incremental con técnicas de modelamiento de ecuaciones estructurales en LISREL (8.8). Este procedimiento, tal y como lo describe Byrne (1998), utiliza un algoritmo que produce estimados de máxima verosimilitud en lugar de mínimos cuadrados. La máxima verosimilitud es, y ha sido, el método más aplicado al análisis de estructuras de covarianza porque tiene la ventaja de mejorar los parámetros estimados para minimizar la función de ajuste (Hair et al., 2007). Bagozzi et al. (1991) sugieren y demuestran que, a diferencia de otras técnicas como la regresión, el AFC es una herramienta adecuada para detectar y analizar el error de medición.

Es de notar que la técnica empleada para analizar la hipótesis planteada es la más utilizada en estudios de equivalencia de medición por ser más precisa que otras posibles alternativas (véase Hair et al., 2006; Wernsing, 2014). Otros métodos de estimación de factores (P. ej. componentes principales) a menudo se consideran preliminares a las técnicas de AFC por modelamiento de ecuaciones estructurales (Hair et al., 2006). El algoritmo de componentes principales, dependiendo de los datos, puede (o no) dar como resultado el número de factores que se han determinado a priori. También, todos los reactivos muestran una carga en todos los factores y su significatividad estadística, que a menudo se determina en base a la magnitud de la carga, no es exacta. En comparación, el AFC por modelamiento de ecuaciones estructurales es más restrictivo pues parte de una indicación exacta sobre el número de factores a obtener y de la forma en que los reactivos cargarán en tales factores. El algoritmo hace que los reactivos carguen solamente en los factores que se indican a priori pudiéndose determinar exactamente si la carga es significativa o no con base en los datos disponibles.

Antes del análisis de invariancia, por cada constructo y por cada país, se llevó a cabo un análisis factorial confirmatorio en forma individual. Estos AFC independientes mostraron si los conceptos de satisfacción laboral y compromiso organizacional existen en las culturas consideradas y si las escalas funcionan adecuadamente cuando se analizan por separado en cada una de ellas. Los resultados de estos análisis son indicativos de la validez convergente de los constructos.

Posterior a los análisis individuales por país, se llevó a cabo el análisis de invariancia de medición para cada constructo con ambas muestras en forma conjunta y simultánea. De acuerdo con varias fuentes (P. ej. Byrne, 1998; Hair et al., 2006), para que una escala de medición se considere equivalente entre dos muestras, la evidencia debe respaldar tres tipos de equivalencia o invariancia de medición: configural, métrica y escalar. Esto es, el constructo analizado debe existir en las muestras de las dos culturas cuando el modelo de medición se prueba simultáneamente en ellas (equivalencia configural). Además, las cargas (o pesos) factoriales de los reactivos en el constructo deben ser equivalentes en las dos muestras cuando se les fuerza a serlo (equivalencia métrica). Finalmente, los interceptos de los reactivos de la escala también deben ser equivalentes en los dos conjuntos de datos (equivalencia escalar). Cada tipo de equivalencia necesita a la anterior como requisito indispensable (P. ej. sin equivalencia métrica no puede haber equivalencia escalar).

Aunque la equivalencia configural es relativamente fácil de entender aun en términos matemáticos, la interpretación conceptual de la equivalencia métrica y escalar es más sencilla de entender que su explicación numérica. Por un lado, cuando las muestras de dos culturas 
son equivalentes en términos métricos, los respondientes de ambas muestras utilizaron los intervalos entre los valores de la escala en forma similar. Recurriendo a una analogía, si la escala de medición bajo análisis fuera un metro, éste equivaldría a la misma longitud en los dos países indicando así que es una medida confiable en el sentido de que no es más grande en un lugar que en otro. Por otro lado, cuando la escala de medición es equivalente en términos escalares, los individuos encuestados atribuyeron el mismo significado a los valores (o puntajes) representados en la escala. En la analogía del metro, si éste no fuera escalarmente equivalente, mientras que en un país una planta de 50 centímetros podría considerarse como "muy alta", en otro podría apreciarse como "alta", a secas. Como se infiere, cuando no hay evidencia de equivalencia escalar, dos personas de diferentes culturas podrían indicar puntajes iguales en una escala de medición aunque las percepciones y los significados fuesen diferentes.

\section{Resultados}

En la Tabla 1 se muestran los estimados de confiabilidad así como algunos estadísticos descriptivos. Aunque el compromiso organizacional parece tener una menor consistencia interna en México que en Estados Unidos, las medidas exhiben niveles de confiabilidad aceptables en ambos países.

Tabla 1

Confiabilidad y estadística descriptiva

\begin{tabular}{|c|c|c|c|c|c|c|c|}
\hline & \multicolumn{2}{|c|}{$\alpha$} & \multicolumn{2}{|c|}{ Media } & \multirow{2}{*}{$t$} & \multicolumn{2}{|c|}{ Desv. est. } \\
\hline & México & EE.UU. & México & EE.UU. & & México & EE.UU. \\
\hline $\begin{array}{l}\text { Satisfacción } \\
\text { laboral }\end{array}$ & 0.90 & 0.89 & 5.32 & 4.71 & $5.68 * * *$ & 1.24 & 1.34 \\
\hline $\begin{array}{l}\text { Compromiso } \\
\text { organizacional }\end{array}$ & 0.70 & 0.84 & 4.40 & 4.24 & 1.80 & 1.06 & 1.12 \\
\hline
\end{tabular}

$* * *=\mathrm{p}<0.001$

Fuente: Elaboración propia

En lo que respecta a los niveles de satisfacción y compromiso, la Tabla 1 indica que ambos son mayores en México que en EE.UU. La diferencia, sin embargo, es significativa sólo en el caso de la satisfacción. Notoriamente, que en México las medias sean más altas y las desviaciones estándar sean menores, hace sospechar que los respondientes mexicanos fueron más proclives que los estadounidenses a los sesgos de condescendencia y lenidad. Esto es, los respondientes mexicanos tendieron más que sus contrapartes estadounidenses a utilizar los valores extremos positivos de la escala (de ahí la media más alta) y a utilizar menos el rango de opciones que se les dio (de aquí la desviación estándar menor). La Tabla 1 muestra que la correlación entre satisfacción y compromiso es relativamente alta en los dos países. Esto concuerda con resultados anteriores sobre la relación entre ambos constructos (Harrison et al., 2006).

La Tabla 2 muestra el resultado del análisis factorial confirmatorio para la satisfacción laboral en forma independiente para cada país. Como se observa, tanto en México como en EE.UU., el AFC sugiere que la matriz de covarianza de los datos originales ajusta muy bien a la 
matriz de covarianza teórica en la cual tales datos cargan en un solo constructo: la satisfacción laboral ( $\chi 2$ no significativa en ambos casos). Aunque el ajuste es ligeramente superior en México (Méx. $\chi 2=3.08<$ EE.UU. $\chi 2=5.76$ ), el CFI y el NNFI apoyan este resultado en ambos países $(>0.98)$. El RMSEA es un tanto marginal para los modelos indicados (peor en EE.UU.); lo cual no es una sorpresa ya que este indicador es inexacto en modelos con muy pocos grados de libertad (gl) (Kenny, Kaniskan y McCoach, 2015) ${ }^{6}$. En resumen, el AFC sugiere que el constructo de satisfacción laboral existe en los datos de ambos países y que la escala funciona razonablemente bien en las dos culturas en forma independiente.

Tabla 2

AFC y análisis de equivalencia de la escala de satisfacción laboral

\begin{tabular}{|c|c|c|c|c|c|c|c|}
\hline Modelo / Indicador & $\chi^{2}$ & $\mathrm{gl}$ & RMSEA & CFI & NNFI & $\chi^{2 / \mathrm{gl}}$ & $\Delta \chi^{2}$ \\
\hline AFC México $(n=287)$ & 3.08 & 2 & 0.04 & 1.00 & 1.00 & 1.54 & \\
\hline AFC Estados Unidos $(\mathrm{n}=273)$ & 5.76 & 2 & 0.08 & 1.00 & 0.98 & 2.88 & \\
\hline $\begin{array}{l}\text { Modelo de equivalencia de estructura } \\
\text { factorial (equivalencia configural) }\end{array}$ & 8.84 & 4 & 0.07 & 1.00 & 0.99 & 2.21 & \\
\hline $\begin{array}{l}\text { Modelo de equivalencia de cargas } \\
\text { factoriales (equivalencia métrica) }\end{array}$ & $16.9 *$ & 8 & 0.06 & 1.00 & 0.99 & 2.11 & 8.06 \\
\hline $\begin{array}{l}\text { Modelo de equivalencia de cargas } \\
\text { factoriales y de interceptos de los reactivos } \\
\text { (equivalencia escalar) }\end{array}$ & $87.63^{* * *}$ & 13 & 0.14 & 0.96 & 0.94 & 6.74 & $70.73^{* * *}$ \\
\hline
\end{tabular}

$*=\mathrm{p}<0.05 ; * *=\mathrm{p}<0.01 ; * * *=\mathrm{p}<0.001$

Fuente: Elaboración propia

Para ahondar más en los resultados de la Tabla 2, la Figura 3 muestra el modelo de medición examinado en ambas muestras en forma independiente. La Tabla 3 exhibe las cargas (o pesos factoriales) obtenidos en cada caso. Estas cargas se presentan en estimados de máxima verosimilitud, y, como se observa en las dos muestras, todas son significativas al nivel de 0.05 sugiriendo que todos los reactivos de la escala forman un solo factor en el que convergen adecuadamente (validez convergente). Es importante notar que tanto en la Tabla 3, como en la Figura 3, se indican los reactivos de la escala como "S" (1 al 5). Se omite presentar las preguntas originales para evitar violaciones a los derechos de autor de los postulantes de la escala. No obstante, los reactivos están disponibles en la fuente citada anteriormente.

\footnotetext{
${ }^{6}$ Los modelos mostrados en las tablas 2 y 3 permiten la covariación libre del error de algunas de las variables observadas (reactivos de la escala). Esto, con base en los índices de modificación reportados por LISREL. Los ajustes son muy pocos y en ningún caso violan la validez convergente y discriminante de los constructos, aunque sí aumentan los parámetros estimados libremente por el modelo y reducen los grados de libertad. Sin embargo, los grados de libertad son muy reducidos aún sin el aumento de los parámetros estimados libremente. La covariación libre del error de algunas de las variables observadas, por lo tanto, no altera significativamente ni el RMSEA ni ninguno de los indicadores globales de ajuste.
} 


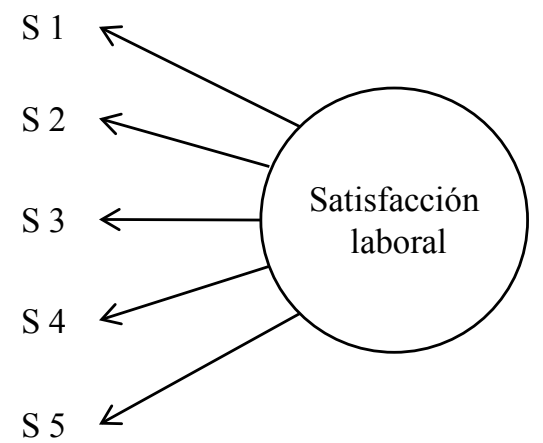

Figura 3. Modelo de medición de la satisfacción laboral Fuente: Elaboración propia

Tabla 3

Cargas factoriales de los reactivos de satisfacción laboral por muestra (puntajes estandarizados)

\begin{tabular}{l|cc}
\multicolumn{3}{c}{ Satisfacción laboral } \\
\hline Reactivos & México & EE.UU. \\
\hline S 1 & $0.79^{*}$ & $0.75^{*}$ \\
S 2 & $0.88^{*}$ & $0.89^{*}$ \\
S 3 & $0.90^{*}$ & $0.95^{*}$ \\
S 4 & $0.77^{*}$ & $0.85^{*}$ \\
S 5 & $0.66^{*}$ & $0.55^{*}$ \\
\hline \multicolumn{2}{l}{}
\end{tabular}

Fuente: Elaboración propia

El resultado de los análisis de equivalencia entre culturas de la escala de satisfacción laboral también se muestra en la Tabla 2. Con respecto a la equivalencia configural, los resultados indican que el modelo de medición funciona bastante bien cuando se fuerza a los datos observados a cargar en el constructo de satisfacción laboral en forma simultánea en ambas muestras $(\chi 2$ no significativa, y CFI y NNFI > 0.99). Este hallazgo confirma los resultados de los AFC independientes por país. En realidad, la escala en cuestión funciona muy similarmente en las culturas analizadas pues en la Tabla 2 también se muestra evidencia de equivalencia métrica aceptable. No sólo el CFI y el NNFI son altos en este caso (>0.99), sino que el indicador $\Delta \chi 2$ que compara el nivel de ajuste de este modelo con el del configural tampoco es significativo. Este resultado sugiere que no hay pérdida de ajuste entre la matriz de covarianza original y la teórica cuando, además de forzar a los datos a cargar en el constructo de satisfacción, también se fuerza a que las cargas (o pesos) factoriales de los reactivos sean equivalentes en las dos muestras. Al parecer, los respondientes mexicanos y sus contrapartes estadounidenses utilizaron los intervalos entre los valores de la escala de satisfacción en forma muy similar.

Desafortunadamente, en la Tabla 2 no se muestra evidencia de equivalencia escalar. El indicador $\Delta \chi^{2}$ que compara el nivel de ajuste del modelo escalar y el métrico es significativo 
siendo el CFI y el NNFI superiores en el segundo. Esto indica que cuando se fuerza a los interceptos de los reactivos a ser equivalentes entre las muestras (en adición a forzar a los reactivos a cargar en un solo constructo y a las cargas factoriales a ser equivalentes), el ajuste del modelo se deteriora significativamente. Este hallazgo implica que los niveles de satisfacción tienen significados diferentes en México y en Estados Unidos. En otras palabras, mientras que para un mexicano un puntaje de cinco podría significar un nivel muy alto de satisfacción, para un estadounidense tal puntaje podría representar un nivel más alto (o más bajo) del mismo concepto.

Con respecto a la escala de compromiso organizacional, la Tabla 4 muestra los resultados de los AFC independientes por país. Como se observa, tanto para México como para EE.UU., los resultados sugieren que el compromiso organizacional existe en los datos analizados. En este caso, no obstante, la evidencia es menos contundente que en el caso de la satisfacción laboral, pues la $\chi^{2}($ Méx. $=27.87 ;$ EE.UU. $=32.60)$ es significativa al nivel de 0.05 en ambos países y el CFI y el NNFI son ligeramente menores a uno. Con respecto al RMSEA, se indica la misma precaución referente a los pocos grados de libertad (gl) del modelo.

La Figura 4 muestra el modelo de medición estimado para el compromiso organizacional. Por su parte, la Tabla 5 indica que las cargas factoriales de los reactivos son significativas y que, por lo tanto, convergieron adecuadamente en un solo factor (el compromiso organizacional) en las dos muestras. Nótese en este caso la misma precaución que en la satisfacción laboral con respecto a los nombres de los reactivos.

Tabla 4

AFC y análisis de equivalencia de la escala de compromiso organizacional

\begin{tabular}{|c|c|c|c|c|c|c|c|}
\hline Modelo / Indicador & $\chi^{2}$ & $\mathrm{gl}$ & RMSEA & CFI & NNFI & $\chi^{2 / \mathrm{gl}}$ & $\Delta \chi^{2}$ \\
\hline AFC México $(n=287)$ & $27.87^{*}$ & 17 & 0.05 & 0.99 & 0.98 & 1.64 & \\
\hline AFC Estados Unidos $(\mathrm{n}=273)$ & $32.60^{*}$ & 17 & 0.06 & 0.99 & 0.98 & 1.92 & \\
\hline $\begin{array}{l}\text { Modelo de equivalencia de estructura } \\
\text { factorial (equivalencia configural) }\end{array}$ & $60.86^{* *}$ & 34 & 0.05 & 0.99 & 0.98 & 1.79 & \\
\hline $\begin{array}{l}\text { Modelo de equivalencia de cargas } \\
\text { factoriales (equivalencia métrica) }\end{array}$ & $141.8^{* * *}$ & 41 & 0.09 & 0.96 & 0.940 & 3.46 & $80.94 * * *$ \\
\hline $\begin{array}{l}\text { Modelo de equivalencia de cargas } \\
\text { factoriales y de interceptos de los reactivos } \\
\text { (equivalencia escalar) }\end{array}$ & $187.29 * * *$ & 49 & 0.10 & 0.94 & 0.930 & 3.82 & $45.49^{* * *}$ \\
\hline
\end{tabular}

Fuente: Elaboración propia 


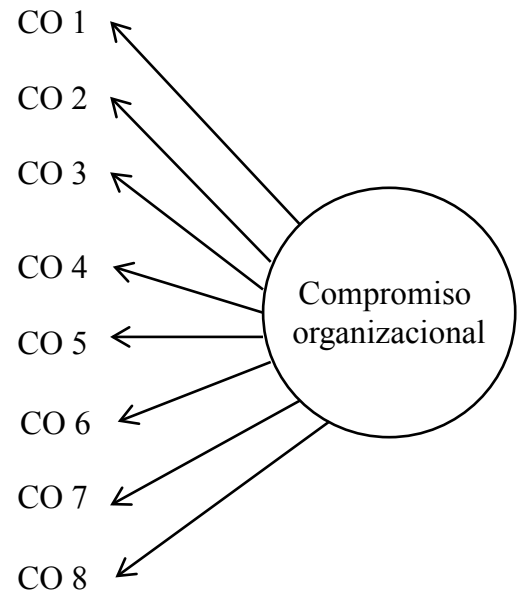

Figura 4. Modelo de medición del compromiso organizacional Fuente: Elaboración propia

Tabla 5

Cargas factoriales de los reactivos de compromiso organizacional por muestra (puntajes estandarizados)

\begin{tabular}{l|cc}
\hline \multicolumn{3}{c}{ Compromiso organizacional } \\
\hline Reactivos & México & EE.UU. \\
\hline CO 1 & $0.69^{*}$ & $0.63^{*}$ \\
CO 2 & $0.88^{*}$ & $0.65^{*}$ \\
CO 3 & $0.61^{*}$ & $0.61^{*}$ \\
CO 4 & $0.31^{*}$ & $0.30^{*}$ \\
CO 5 & $0.29^{*}$ & $0.53^{*}$ \\
CO 6 & $0.33^{*}$ & $0.74^{*}$ \\
CO 7 & $0.76^{*}$ & $0.78^{*}$ \\
CO 8 & $0.29^{*}$ & $0.58^{*}$ \\
\hline \multirow{2}{*}{$=p<0.05$} & &
\end{tabular}

Fuente: Elaboración propia

El resultado de los análisis de equivalencia configural y métrica de la escala de compromiso también se exhibe en la Tabla 4. Como se observa, la equivalencia configural de la escala es aceptable debido a que el CFI y el NNFI son altos en este caso. Esto, a pesar de que la $\chi^{2}$ sea significativa; lo cual se explica porque el cálculo se basa en el total de los datos combinados $(\mathrm{N}=560)$ y el indicador en cuestión es muy sensible al incremento en el tamaño de la muestra. No obstante, y a diferencia de la satisfacción laboral, en la Tabla 4 no se muestra evidencia de equivalencia métrica ( $\Delta \chi^{2}$ es significativo siendo $\chi 2$, CFI y NNFI superiores en el modelo configural). En otras palabras, la gente de México y la de EE.UU. parecen haber utilizado los intervalos entre los valores de la escala en forma distinta. 
Con respecto a la equivalencia escalar, la Tabla 4 indica que los puntajes asociados a la escala de compromiso organizacional tampoco son equivalentes entre los países. El análisis de equivalencia en mención arroja un $\Delta \chi 2$ significativo indicando un deterioro en los índices globales de bondad de ajuste en el modelo escalar en comparación con el métrico.

En resumen, la evidencia indica que mientras que la escala de satisfacción laboral es equivalente en dos aspectos (configural y métrico) entre México y Estados Unidos, la de compromiso organizacional sólo es en uno (configural). En otras palabras, los resultados sugieren que los constructos que se examinaron existen en ambas culturas aunque la gente utiliza la escala de medición en forma similar sólo en el caso de la satisfacción. Sin embargo, dado que en ambos casos no se encontró evidencia de equivalencia escalar, la comparación directa de los niveles de los constructos (medias) es inexacta pues las cantidades de satisfacción laboral y compromiso organizacional capturadas en los puntajes de las escalas no parecen significar lo mismo en la cultura mexicana que en la estadounidense.

Con base en lo anterior, se acepta la hipótesis planteada. Esto es, que las escalas analizadas no son completamente equivalentes entre los países considerados. En consecuencia, aunque el uso independiente de las escalas en ambas culturas podría ser adecuado, la comparación transcultural directa de los niveles de satisfacción y compromiso puede ser tentativa pero no concluyente. Dicho esto, nótese que la diferencia en los niveles de satisfacción entre México y EE.UU. mostrada en la Tabla 1, aunque es ilustrativa, no es confiable a pesar del nivel de significatividad del estadístico $t$.

\section{Discusión}

El propósito de este trabajo fue el de analizar la equivalencia de una escala de satisfacción laboral y otra de compromiso organizacional entre México y Estados Unidos. Para tal efecto, se encuestó a estudiantes de maestría en administración en los dos países. El resultado de los análisis indica que las escalas no son equivalentes entre las naciones abordadas. Este hallazgo tiene implicaciones para los estudios cuyo propósito es medir y comparar los niveles de satisfacción y compromiso en contextos internacionales.

Los hallazgos mostrados en este documento indican que la comparación transcultural de ciertos fenómenos puede ser inexacta. La satisfacción laboral y el compromiso organizacional son conceptos acuñados en Estados Unidos. En consecuencia, las escalas para medirlos también lo son. Esto es interesante a la luz de los estudios de la cultura donde se tiene por entendido que las teorías, conceptos y metodologías científicas conllevan la cosmovisión y las creencias y valores de las sociedades en las que emergen (Scott y Davis, 2007). Por implicación, los conceptos de satisfacción laboral y compromiso organizacional podrían no existir en sociedades distintas a la estadounidense (o al menos no en la misma forma). También, es posible que las escalas de medición que se utilizan para abordar empíricamente tales fenómenos no sean equivalentes en otras sociedades aun cuando se empleen métodos que afiancen la equivalencia lingüística. En concordancia con otros estudios (P. ej. Peterson, Puia y Suess, 2003), los resultados de este trabajo indican que tanto la satisfacción laboral como el compromiso organizacional existen en México y en su vecino del norte. No obstante, los resultados también extienden este conocimiento pues muestran que, aunque los conceptos existen, las escalas de medición no son equivalentes entre las culturas. 
Los hallazgos de esta investigación son inéditos pues no existe evidencia previa de la equivalencia de medición de escalas de satisfacción laboral y compromiso organizacional entre México y EE.UU. Notablemente, la evidencia presentada en este documento hace eco a otras obras anteriores que han mostrado que las escalas para medir constructos psicológicos como resiliencia, esperanza, auto-eficacia y optimismo (Wernsing, 2014), así como el bienestar subjetivo (Xiaoming, et al., 2015), podrían no ser equivalentes entre culturas. Este estudio también responde a la preocupación general en la investigación sobre el control del error sistemático de medición cuando una escala se aplica en más de una cultura con fines de comparación de medias (Aguinis y Edwards, 2014).

La falta de equivalencia de las escalas de medición de satisfacción y compromiso implica una precaución, aunque no necesariamente una limitación, para los estudios transculturales. Como se explicó anteriormente, cuando una escala funciona desigualmente a través de dos (o más) culturas, la comparación de los niveles de los conceptos involucrados es ilustrativa pero no confiable. No obstante, esto no quiere decir que los estudios transculturales son inapropiados o imposibles. Existen formas estadísticas confiables para remover los sesgos y distorsiones que pueden alterar las respuestas de los participantes (P. ej. condescendencia y lenidad). Uno de tales métodos es el empleado por Hosftede et al. (2010), cuyos afamados estudios son precisamente una comparación directa de los niveles de las dimensiones culturales a través de una cantidad considerable de países. También hay que aclarar que cuando los estudios transculturales tienen como objetivo analizar correlaciones (en lugar de diferencias de medias), no necesariamente se necesita procesar los datos para que las puntuaciones equivalgan a significados similares a través de las culturas (equivalencia escalar). De acuerdo con Hair et al. (2006) la equivalencia métrica (que un metro mida lo mismo en varios países) sería suficiente en los estudios correlacionales.

Ciertamente, este estudio presenta varias limitaciones que restringen la generalización de los resultados. Como en todas las investigaciones que involucran la traducción de una escala, es posible que los problemas de equivalencia que se detectaron se deban a errores de traducción. Esta posibilidad siempre está latente aunque el proceso haya sido cuidadoso y diligente. También, los resultados aquí mostrados tienen problemas de validez ecológica. En otras palabras, las diferencias encontradas podrían deberse a diferencias en la cultura de las organizaciones (universidades) en donde se recolectaron los datos en lugar de diferencias en la cultura de los países. Sin embargo, esta posibilidad es moderada debido a que, a mayor o menor grado, la cultura organizacional refleja la cultura nacional (Gerhart, 2009). En este tenor, se implica que los problemas de equivalencia de las escalas involucradas podrían deberse a factores culturales. Esto, sin embargo, se presume pero no se demuestra ni se analiza.

Las limitaciones anteriores representan áreas futuras de investigación. Por ejemplo, recolectar datos en diferentes regiones de ambos países, así como analizar muestras un poco más grandes, ayudaría a entender mejor cómo las diferencias culturales entre México y EE.UU. afectan el uso de las escalas de medición y la comparación directa de resultados de satisfacción laboral, compromiso organizacional, y otras actitudes referentes al trabajo como el involucramiento y el enganchamiento (engagement). No hay que olvidar que empresarios y ejecutivos de negocios transnacionales están interesados en el tema ya que se confía en que las actitudes hacia (y en) el trabajo afectan significativamente la productividad y el desempeño de los empleados. 
Finalmente, el contenido de este documento es una pieza de información cuyo valor estriba en dejar latente las precauciones que deben tomarse en el estudio de la satisfacción laboral y el compromiso organizacional en contextos internacionales.

\section{Conclusiones}

La satisfacción laboral y el compromiso organizacional son, muy probablemente, los antecedentes más estudiados del comportamiento humano en el trabajo. Dadas sus implicaciones críticas en la efectividad organizacional, el estudio de tales conceptos se ha extendido al mundo desde su origen en Estados Unidos (principalmente). En paralelo, también se han adoptado las formas (escalas) de medición sin, en muchos casos, primero identificar si éstas funcionan similarmente en contextos culturales diferentes. Esta identificación es importante ya que, si las escalas no son equivalentes entre países, un resultado que indique que las medias de satisfacción (o compromiso) difieren (o no) entre ellos, sería dudoso puesto que la posibilidad existe de que tales diferencias sean producto del uso particular que las personas de distintos países dan a tales escalas. En este sentido, los hallazgos de este trabajo de investigación indican que las escalas analizadas no son equivalentes entre México y su vecino del norte. Por lo tanto, antes de proceder a realizar comparaciones directas de los niveles de tales constructos entre estos países, los investigadores deben tomar precauciones en la preparación, uso y análisis de los datos. En la medida en que se tomen precauciones a este respecto, los resultados serán más confiables y las comparaciones internacionales más precisas. Al final, estudios confiables y precisos contribuyen a conocer más los fascinantes efectos y matices de la satisfacción laboral y el compromiso organizacional en entornos internacionales.

\section{Referencias}

Aguinis, H. y Edwards, J. (2014). Methodological wishes for the next decade and how to make wishes come true. Journal of Management Studies, 51 (1), 143-174. https://doi.org/10.1111/joms.12058

Allen, N. y Meyer, J. P. (1990). The measurement and antecedents of affective, continuance and normative commitment to the organization. Journal of Occupational Psychology, 63, 1-18. https://doi.org/10.1111/j.2044-8325.1990. tb00506.x

Allen, N. y Meyer, J. P. (1996). Affective, continuance, and normative commitment to the organization: An examination of construct validity. Journal of Vocational Behavior, 49, 252-276. https://doi.org/10.1006/jvbe.1996.0043

Bagozzi, R. y Yi, Y. (1991). Multitrait-multhimethod matrices in consumer research. Journal of Consumer Research, 17, 426-439. https://doi.org/10.1207/s15327663jcp0202_03

Bagozzi, R., Yi, Y. y Phillips, L. (1991). Assessing construct validity in organizational research. Administrative Science Quarterly, 36, 421-458. https://doi.org/10.2307/2393203

Bowling, N., Hendricks, E. y Wagner, S. (2008). Positive and negative affectivity and facet satisfaction: A meta-analysis. Journal of Business Psychology, 23, 115-125. https://doi.org/10.1037/e518612013-334

Brayfield. A. H. y Rothe, H. (1951). An index of job satisfaction. Journal of Applied Psychology, 35, 207-311. https:// doi.org/10.1037/t08600-000

Burrell, G. y Morgan, G. (1979). Sociological Paradigms and Organizational Analysis. (1a ed.). Inglaterra: Ashgate Publising Limited.

Byrne, B. (1998). Structural Equation Modeling with LISREL, PRELIS, and SIMPLIS. (1a ed.). EE.UU.: Lawrence Erlbaum Associates.

Campbell, D. y Stanley, J. (1963). Experimental and Quasi-experimental Designs for Research. (1a ed.). EE.UU.: Houghton Mifflin Company. 
Coatsworth, J. y Rico, C. (1989). Imágenes de México en Estados Unidos. (1a ed.). México: Fondo de Cultura Económica.

Cook, T., Campbell, D., y Perachio, L. (1990). Quasiexperimentation. En M.D. Dunnette y L.M. Hough (Eds.), Handbook of Industrial and Organizational Psychology. (pp. 491-576). EE.UU.: Palo Alto Consulting Psychologists.

Curry, J. P., Wakefield, D. S., Price, J. L., y Mueller, C. W. (1986). On the causal ordering of job satisfaction and organizational commitment. Academy of Management Journal, 29, 847-858. https://doi.org/10.2307/255951

Etzioni, A. (1961). Comparative Analysis of Complex Organizations. (1a ed.). EE.UU.: Free Press.

Festinger, L. (1957). A Theory of Cognitive Dissonance. EE.UU.: Stanford University Press.

Fiske, D. (1982). Convergent-discriminant validation in measurements and research strategies. En Brinbirg, D. y Kidder, H. (Eds.), Forms of Validity in Research. (pp.77-92). EE.UU.: Jossey-Bass.

Gazol Sánchez,A. (2010). Una unión aduanera en América del Norte ¿Es conveniente? ¿Es posible? Economía UNAM, 7 (19), 45-68.

Gerhart, B. (2009). How much does national culture constrain organizational culture? Management and Organization Review, 5 (2), 241-259. https://doi.org/10.1111/j.1740-8784.2008.00117.x

Gill, H., Meyer, J. P., Lee, K., Shin, K. y Yoon, C. (2011). Affective and continuance commitment and their relations with deviant workplace behaviors in Korea. Asia Pacific Journal of Management, 28, 595-607. https://doi. org/10.1007/s10490-009-9165-0

Gillespie, M., Balzer, W., Brodke, M., Garza, M. Gerbec, E., Gillespie, J., Gopalkrishnan, P., Lengyel, J., Sliter, K., Sliter, M., Withrow, S. y Yugo J. (2016). Normative measurement of job satisfaction in the U.S. Journal of Managerial Psychology, 31 (2), 516-536. https://doi.org/10.1108/jmp-07-2014-0223

Hair, J., Black, W., Babin, B., Anderson, R. y Tatham, R. (2006). Multivariate Data Analysis. (6a ed.). EE.UU.: Pearson Prentice-Hall.

Harrison, D., Newman, D. y Roth, P. (2006). How important are job attitudes? Meta-analytic comparisons of integrative behavioral outcomes and time sequences. Academy of Management Journal, 49 (2), 305-325. https://doi. org/10.5465/amj.2006.20786077

Hofstede, G. (1980). Culture's Consequences: International Differences in Work-Related Values. (1a ed.). EE.UU.: Sage Publications.

Hofstede, G., Hofstede, G, J. y Minkov, M. (2010). Cultures and Organizations. Software of the Mind. (3a ed.). EE.UU.: McGrawHill.

House, R., Hanges, P., Javidan, M., Dorfman, P. y Gupta, V. (2004). Culture, Leadership and Societies. The GLOBE Study of 62 Societies. (1a ed.). EE.UU.: SAGE Publications.

Iaffaldano, M. y Muchinsky, P. (1985). Job satisfaction and job performance: A meta-analysis. Psychological Bulletin, 97, 251-273. https://doi.org/10.1037//0033-2909.97.2.251

Judge, T., Bono, J. y Locke, E. (2000). Personality and job satisfaction: The mediating role of job characteristics. Journal of Applied Psychology, 85, 237-249. https://doi.org/10.1037//0021-9010.85.2.237

Judge, T. y Klinger, R. (2008). Job Satisfaction. Subjective well-being at work. En Eid M. y Larsen, R. (Eds.), The Science of Subjective Well-Being. (pp. 393-413). EE.UU.: The Gilford Press.

Judge, T., Thoresen, C., Bono, J. y Patton, G. (2001). The job satisfaction-job performance relationship: A qualitative and quantitative review. Psychological Bulletin, 127 (3), 376-407. https://doi.org/10.1037//0033-2909.127.3.376

Kenny, D., Kaniskan, B. y McCoach, B. (2015). The performance of RMSEA in models with small degrees of freedom. Sociological Methods \& Research, 44 (3), 486-507. https://doi.org/10.1177/0049124114543236

Kogut, B. y Singh, H. (1988). The effect of national culture on the choice of entry mode. Journal of International Business Studies, 19 (3), 411-432. https://doi.org/10.1057/palgrave.jibs.8490394

Landy, F. (1989). Psychology of Work Behavior. (4a ed.). EE.UU.: Brooks/Cole Pub Co.

Locke, E. (1969). What is job satisfaction? Organizational Behavior and Human Performance, 4, 309-226. https://doi. org/10.1016/0030-5073(69)90013-0

Locke, E. (1986). Generalizing from Laboratory to Field Settings: Research Findings from Industrial-Organizational Psychology, Organizational Behavior, and Human Resource Management. Estados Unidos: Lexington Books. 
Marín, G., Gamba, R. y Marín, B. (1992). Extreme response style and acquiescence among hispanics. The role of acculturation and education. Journal of Cross-Cultural Psychology, 23 (4), 498-509. https://doi. org/10.1177/0022022192234006

McSweeney, B. (2002). Hofstede's model of national cultural differences and the consequences: A triumph of faith -a failure of analysis. Human Relations, 55, 89-118. https://doi.org/10.1177/0018726702055001602

Meyer, J. P., Allen, N. y Smith, C. (1993). Commitment to organizations and occupations: Extension and test of a three-component conceptualization. Journal of Applied Psychology, 78 (4), 538-551. https://doi.org/10.1037//00219010.78.4.538

Meyer, J.P., Stanley, D., Herscovitch, L. y Topolnytsky, L. (2001). Affective, continuance, and normative commitment to the organization: A meta-analysis of antecedents, correlates, and consequences. Journal of Vocational Behavior, 61, 20-52. https://doi.org/10.1006/jvbe.2001.1842

Minkov, M. y Hofstede, G. (2012). Is national culture a meaningful concept? Cultural values delineate homogeneous national clusters on in-country regions. Cross-Cultural Research, 42 (2), 133-159. https://doi. org $/ 10.1177 / 1069397111427262$

O’Connor, E., Peters, L. y Gordon, S. (1978). The measurement of job satisfaction: Current practices and future considerations. Journal of Management, 4 (2), 17-26. https://doi.org/10.1177/014920637800400202

Paz, O. (2009). El Laberinto de la Soledad. Postdata, Vuelta al Laberinto de la Soledad. (4a ed.). México: Fondo de Cultura Económica.

Peterson, C., Park, N., Hall, N. y Seligman, M. (2009). Zest and work. Journal of Organizational Behavior, 30, 161172. https://doi.org/10.1002/job.584

Peterson, D., Puia, G. y Suess, F. (2003). "Yo tengo la camiseta (I have the shirt on)": An exploration of job satisfaction and commitment among workers in Mexico. Journal of Leadership and Organizational Studies, 10 (2), 73-88. https://doi.org/10.1177/107179190301000208

Podsakoff, P., MacKenzie, S., Lee, J. y Podsakoff, N. (2003). Common method biases in behavioral research: A critical review of the literature and recommended remedies. Journal of Applied Psychology, 88 (5), 879-903. https://doi. org/10.1037/0021-9010.88.5.879

Podsakoff, P. y Organ, D. (1986). Self-reports in organizational research: Problems and prospects. Journal of Management, 12, 69-82. https://doi.org/10.1177/014920638601200408

Porter, L., Mowday, R., y Steers, R. (1982). Employee-organizational Linkages: The Psychology of Commitment, Absenteeism, and Turnover. (1a ed.). EE.UU.: Academic Press.

Porter, L., Steers, R. y Mowday, R. (2007). Do employee attitudes toward organizations matter? The study of employee commitment to organizations. En Smith, K. y Hitt, M. (Eds.), Great Minds in Management. The Process of Theory Construction. (pp. 171-189). EE.UU.: Oxford University Press.

Raina, R. y Britt Roebuck, D. (2016). Exploring cultural influence on managerial communication in relationship to job satisfaction, organizational commitment, and the employees' propensity to leave in the insurance sector of India. International Journal of Business Communication, 53 (1), 97-130. https://doi.org/10.1177/2329488414525453

Rhoades, L. y Eisenberger, R. (2002). Perceived organizational support: A review of the literature. Journal of Applied Psychology, 87 (4), 698-714. https://doi.org/10.1037//0021-9010.87.4.698

Riding, A. (1985). Vecinos Distantes. Un Retrato de los Mexicanos. (1a ed.). México: Planeta.

Ruder, M. y Bless, H. (2003). Mood and the reliance on the ease of retrieval heuristic. Journal of Personality and Social Psychology, 85 (1), 20-32. https://doi.org/10.1037/0022-3514.85.1.20

Salanova, M., Martínez, I. y Llorens, S. (2005). Psicología organizacional positiva. En Palací Descals, F. J. (Coord.), Psicología de la Organización. (pp. 349-376). España: Pearson-Prentice Hall.

Scott, R. y Davis, G. (2007). Organizations and Organizing. Rational, Natural, and Open System Perspectives. EE.UU.: Pearson Education.

Smith, P. (2004). Acquiescent response bias as an aspect of cultural communication style. Journal of Cross-Cultural Psychology, 35 (1), 50-61. https://doi.org/10.1177/0022022103260380

Solinger, O., Olffen, W. y Roe, R. (2008). Beyond the three component model of organizational commitment. Journal of Applied Psychology, 93 (1), 70-83. https://doi.org/10.1037/0021-9010.93.1.70 
Vandenberghe, C., Bentein, K. y Panaccio, A. (2014). Affective commitment to organizations and supervisors and turnover: A role theory perspective. Journal of Management, 12, 1-28. https://doi.org/10.1177/0149206314559779

Vroom, (1964). Work and Motivation. (1a ed.). EE.UU.: Wiley.

Wernsing, T. (2014). Psychological capital: A test of measurement invariance across 12 national cultures. Journal of Leadership and Organizational Studies, 2 (2), 79-190. https://doi.org/10.1177/1548051813515924

Williamson, D. (2002). Forward from a critique of Hofstede's model of national culture. Human Relations, 55 (11), 1373-1395. https://doi.org/10.1177/00187267025511006

Wright, T. y Staw, B. (1999). Affect and favorable work outcomes: Two longitudinal tests of the happy-productive worker thesis. Journal of Organizational Behavior, 20, 1-23. https://doi.org/10.1002/(sici) 1099-1379(199901)20:1<1::aid-job885>3.0.co;2-w

Xiaoming, Z., Weichun, Z., Haixia, Z. y Chi, Z. (2015). Employee well-being in organizations: theoretical model, scale development, and cross-cultural validation. Journal of Organizational Behavior, 36 (5), 621-644. https://doi. org/10.1002/job.1990 